\title{
Is Conceptual Combination Influenced by Word Order?
}

\author{
Phil Maguire \\ Department of Computer Science \\ University College Dublin \\ Belfield, Dublin 4, Ireland \\ Phil.Maguire@ucd.ie
}

\author{
Arthur Cater \\ Department of Computer Science \\ University College Dublin \\ Belfield, Dublin 4, Ireland \\ Arthur.Cater@ucd.ie
}

\begin{abstract}
We describe two experiments using French noun-noun combinations which parallel a study carried out by Gagné (2001) using English combinations. The order of the modifier and head noun are reversed in French, allowing us to investigate whether the influence of relation priming that Gagné found is due to the order of the modifier and head noun or whether it is due to their different functional roles. While our findings indicate that interpretation is influenced by previous exposure to combinations incorporating one of the same constituent nouns, the results show that primes with the same modifier have a greater influence when associated with a different relation to the target. This pattern of influence is similar to that found in English and suggests that the modifier is exclusively involved in relation selection, irrespective of its order in a combination.
\end{abstract}

\section{Introduction}

The combination of two existing words is a productive strategy used by speakers to convey new concepts and extend the limits of the vernacular. The process of understanding these novel compounds is worthy of study, both because it is intimately associated with the creativity of language use and because it provides a constrained domain in which to test cognitive theories of conceptual representation and language comprehension. In English compounds, the first word or modifier attaches further meaning to the second word or head, thus creating a reference to the intended concept. In order to interpret a nominal compound such as "mountain stream", people must find a relation to link the compound's head and modifier. Several different theories have been proposed as to how people find the correct relation with which to link the constituent nouns. Gagné and Shoben's (1997) Competition Among Relations In Nominals (CARIN) theory maintains that there is a fixed, relatively small taxonomy of standard relations that can be used to link the modifier and head noun concepts. According to this theory, the representation of the modifier concept includes statistical knowledge about those relations with which the modifier tends to be used during conceptual combinations. The most available standard relation is the one most frequently used to interpret other compounds containing that same modifier. For instance, the modifier "mountain" is most often associated with the <head LOCATED modifier> relation thus making the combination "mountain stream" easier to interpret than "mountain magazine" which uses the <head ABOUT modifier> relation.

Important evidence in support of the CARIN model is the finding that the modifier's relational distribution influences the ease with which a combined concept can be interpreted. Gagné and Shoben (1997) found that combinations involving a relation used frequently with the modifier were easier to interpret than combinations involving a less frequent relation, while the frequency distribution of the head noun had no influence. This raises the question as to why it should be the case that the frequencies of relations associated with the modifier affect ease of interpretation, but not those of the head noun. Gagné and Shoben (1997) suggest that the modifier may have more of an influence than the head noun because it is encountered first and consequently highly frequent relations for the modifier may become activated prior to frequent relations for the head noun. A second possibility they suggest is that the modifier noun has certain associated properties which give it a semantic privilege in determining the meaning of a combination. One way to test both of these hypotheses is to examine the interpretation of combinations in a language in which the order of the nouns is the reverse of that in English. We adopt such an approach by examining the interpretation of combinations in the French language in order to determine which of the above possibilities can account for Gagné and Shoben's findings.

The following experiments parallel a speeded sensibility study by Gagné (2001) which investigated the ways in which recent exposure to a similar combination influences the processing of a 
subsequent combination. Gagné found that when the prime and the target had the same head noun, there was no significant difference in reaction times between the cases where they shared the same relation and cases where they did not. However, when the modifier was the repeated constituent, primes that used the same relation exerted more influence than those that used a different relation. Thus, "mountain stream" was more effective than "mountain magazine" at priming "mountain goat" while "kitchen chair" and "wood chair" were equally effective at priming "garden chair". Gagné concluded that when the prime and target share the same modifier, relation priming increases the availability of a selected relation within the modifier's relational distribution. We replicate Gagné's study in French in order to determine whether the same effect will be observed.

While conceptual combination in the English language involves the straightforward juxtaposition of two nouns, combinations in French are made up of three separate elements, namely the head, the modifier and a linking preposition. The preposition gives some indication of the relation between the two concepts as different prepositions are used with different relations. The three French prepositions typically used are "de", “à" and "en". While the use of a preposition in French can bias the selection of a particular relation, we have controlled for this by choosing materials exclusively associated with the "de" preposition, which can be used with almost all relations. Consequently this eliminates any alternative influences on relation selection other than those exerted by the modifier and the head.

\section{Empirical Study}

Two separate experiments were carried out. In the first, the prime had the same head as the target and in the second, the modifier was the repeated constituent. In both experiments, there were three conditions. In one condition the prime used the same relation as the target; in another it used a different relation. There was also a neutral condition in which the target combination was preceded by a combination with no common noun constituent. The experimental design follows that of Gagné (2001) and facilitates the analysis of the relative amounts of priming derived from a combination containing the same head or the same modifier as the target. Priming was evaluated by comparing each of the first two conditions with the neutral condition and by comparing response times to target combinations in the same-relation condition with response times to target combinations in the different-relation condition.

\subsection{Method}

Materials. In both experiments, sixty combined concepts were created as targets. For each target combination, three prime combinations were constructed. One used the same relation as the target and either the same head (experiment 1) or the same modifier (experiment 2). Similarly, another combination used a different relation. The control combination shared no noun constituent with the target. Three lists of stimuli were arranged such that there was an equal number of each prime type in each list. Across all three lists, each target was seen with each type of prime combination. Our materials were controlled for plausibility and familiarity. Two raters scored the plausibility and familiarity of the referents of the prime combinations on a Likert scale from 1 to 7 . A twosided Wilcoxon signed-ranks test revealed no reliable differences between conditions for plausibility, familiarity or average syllable length $(p>0.05)$.

Procedure. Each participant was exposed to one of the lists and hence saw each target item only once. The pairs of prime and target items were presented in a randomised order along with 60 filler pairs and the complete set of filler pairs was presented to each individual. Participants sat in front of a computer screen and placed the index finger of their left hand on the $\mathbf{F}$ key of the keyboard and the index finger of their right hand on the $\mathbf{J}$ key. Participants were told that $\mathbf{J}$ corresponded to "Juste" and $\mathbf{F}$ corresponded to "Faux". Trial presentation was self-paced. Following exposure to the prime combination, participants indicated whether it had a sensible, literal interpretation by pressing the appropriate key. Subsequently, the target combination was similarly displayed and participants made another sensibility judgment. There was nothing in the method of presentation to suggest any connection between consecutive combinations.

Participants. 36 native French speakers participated, 18 in each experiment (ages 20-31, M $=24.2$ ). This selection consisted of students and teachers based in Ireland.

\subsection{Results and Discussion}

$9.1 \%$ of trials were excluded from the analysis. $0.8 \%$ of trials were rejected because participants pressed a key other than $\mathbf{J}$ or $\mathbf{F}$. Additionally, 4.6\% of trials were excluded in cases where the response "faux" was incorrectly given. Responses deemed unreasonably fast $(<400 \mathrm{~ms} ; 0.2 \%)$ and unreasonably slow (> $4000 \mathrm{~ms} ; 0.9 \%$ ) were also excluded. After eliminating all trials which did not meet the above criteria, any response times which 
were more than three standard deviations outside each participant's mean were also rejected. This eliminated another $2.6 \%$ of responses. A repeated measures ANOVA test was conducted to examine the effect of prime type on sense-nonsense judgments for each experiment. Tables 1 and 2 display the response time (in milliseconds) for appropriate responses to the target combinations in each of the experiments.

\begin{tabular}{cccc}
\hline & Prime & & $\begin{array}{c}\text { Target } \\
\text { Response }\end{array}$ \\
\cline { 1 - 3 } Same & Same & Same & \\
Time $(m s)$ \\
Mead & Modifier & Relation & \\
$\checkmark$ & $x$ & $\checkmark$ & 994 \\
$\checkmark$ & $x$ & $x$ & 999 \\
$x$ & $x$ & NA $^{1}$ & 1153 \\
\hline
\end{tabular}

Table 1: Response Times (in milliseconds) for Target Combinations in Experiment 1

\begin{tabular}{cccc}
\hline & Prime & & $\begin{array}{c}\text { Target } \\
\text { Response } \\
\text { Time }(m s)\end{array}$ \\
\cline { 1 - 3 } Same & Same & Same & \\
Mead & Modifier & Relation & \\
\cline { 1 - 3 }$x$ & $\checkmark$ & $\checkmark$ & 998 \\
$x$ & $\checkmark$ & $x$ & 1043 \\
$x$ & $x$ & NA & 1062 \\
\hline
\end{tabular}

Table 2: Response Times (in milliseconds) for Target Combinations in Experiment 2

Evidence of priming. Responses to the target combination were faster when the prime and target shared a constituent noun. In the first experiment, the $159 \mathrm{~ms}$ difference between the same-relation and neutral conditions was reliable, $F_{\text {subject }}(1,34)=$ $31.70, p<.01 ; F_{\text {item }}(1,118)=27.30, p<.01$. The $154 \mathrm{~ms}$ difference between the different-relation and neutral conditions was also reliable, $F_{\text {subject }}(1$, $34)=22.22, p<.01 ; F_{\text {item }}(1,118)=27.309, p<$ .01 . In the second experiment the $64 \mathrm{~ms}$ difference between the same-relation and neutral conditions was reliable, $F_{\text {subject }}(2,34)=9.248, p<.05 ; F_{\text {item }}(2$, $118)=11.437, p<.05$. However, the $19 \mathrm{~ms}$ difference between the different-relation and neutral conditions was not reliable, $F_{\text {subject }}(2,34)=$ $.587, p>.05 ; F_{\text {item }}(2,118)=.337, p>.05$.

Relation influence. As predicted by the CARIN theory, the first experiment, in which the head was the repeated constituent, revealed no evidence of relation influence. No significant difference was found between response times to target

\footnotetext{
${ }^{1}$ The relation of the neutral condition was considered irrelevant following Gagné's (2001) finding that priming does not occur when the preceding combination does not share either of the target's constituent nouns
}

combinations in the same-relation and in the neutral conditions. The $5 \mathrm{~ms}$ difference between the two conditions was not reliable $(F \mathrm{~s}<1)$. However, in the repeated modifier experiment the target was easier to interpret when it was preceded by a combination with the same relation than when it was preceded by one with a different relation. Participants responded to targets following the same relation prime $45 \mathrm{~ms}$ quicker than they did to targets following the different relation prime, $F_{\text {subject }}(2,34)=4.349, \quad p<.05 ; F_{\text {item }}(2,118)=$ $4.194, p<.05$. These data indicate that French speakers are only sensitive to relational information associated with the modifier.

Summary. The results of the two experiments show that the influence of a recently viewed combination is affected by its relation only in cases where the target shares the same modifier (experiment 2) and not in cases where it shares the same head (experiment 1). Thus "ruisseau de montagne" (mountain stream) was more effective than "chaussures de montagne" (mountain shoes) at priming "glacier de montagne" (mountain glacier) while "sac de voyage" (travel bag) and "sac de cuir" (leather bag) were equally effective at priming "sac de sport" (sports bag). These results are similar to those of Gagné (2001) and are thus consistent with research in the English language indicating that relational information is associated with the modifier and not with the head noun. Since these effects have been replicated in a language in which the order of the modifier and head are reversed, this suggests that modifiers and head nouns maintain the same role in the process of interpretation regardless of the order in which they are realised. Our findings confirm that relational information is a tangible feature of conceptual combinations and that the association between the modifier and the relation is an intrinsic property that is evident regardless of the order of the constituent nouns.

\section{General Discussion}

While our results correspond with those of Gagné (2001), we interpret them differently. In her study Gagné distinguished two priming effects, namely lexical priming and relation priming. She claimed that when the head noun was repeated, only lexical priming was observed but that when the modifier was repeated, both types of priming were evident. This distinction is not necessary. It is simpler to suppose that the repeated-modifier different-relation condition exhibits an interference effect which diminishes the effectiveness of lexical priming. Such an interference could arise for two reasons, neither of which requires an assumption of 
relation priming. The first possibility is that combinations using a different relation elicit no priming because a different sense of the modifier is associated with each relation. For example, the French term "en chocolat" (made of chocolate) has very different connotations to "à chocolat" (for chocolate) or "de chocolat" (of chocolate). While these terms employ the same modifier, they each have different meanings since the preposition immediately elucidates the modifying capacity of the noun. Though the relation associated with a modifier in English may not be expressed in the same way, the conceptual disparity is likely to persist nonetheless. It is therefore conceivable that the relation with which the modifier is associated can change its meaning and as a result, one modifier might not necessarily prime a combination using the same modifier in a different sense.

A second possibility is that the availability of one meaning of a modifier is increased after encountering a prime using it with that sense. When the same modifier is encountered being used with a different sense in the target, the original sense is more accessible than the appropriate one. Hence, following the prime "sel de mer" (sea salt), participants may find it more difficult to interpret "mal de mer" (sea sickness) because they are more likely to assume the "from the sea" sense of the modifier instead of the correct "caused by the sea" interpretation. An explanation of our results may be due to a combination of the above possibilities, both of which emphasise the co-dependence of the modifier and its associated relation.

While our results have emphasised the link between modifier and relation, they do not suggest that modifier relational frequency is the only factor involved in selecting a plausible relation and it is likely that both the head and the modifier are involved in this process. In order to develop an accurate computational model of conceptual combination, future studies will need to consider the influence of other contributing factors. Certain heads and modifiers are strongly biased towards suggesting one particular relation. For instance, modifiers denoting substances are biased towards the <head MADE OF modifier> relation (e.g. "plastic") and in the same way, head nouns with a strongly associated schema, such as "factory", can be biased towards suggesting a certain relation. Furthermore, relation likelihood may be influenced by the presence of facilitating features (Devereux \& Costello, 2004). Facilitating features are those features of a pair of concepts that are necessary for a given relation to be possible. For example a compound with the modifier "kitchen" is unlikely to be interpreted using the <head MADE OF modifier $>$ relation since kitchens are not a type of substance. Computational models of conceptual combination may have to account for the characteristics of heads and modifiers individually in order to simulate the ways in which each constituent influences relation selection.

\section{Conclusion}

In summary, we investigated the influence of relation priming on the interpretation of French noun-noun compounds in order to ascertain whether the influence of the modifier observed in studies of English stems from its functional properties rather than the fact that it is encountered first. Our results showed that same and differentrelation primes were equally effective when they shared the same head as the target, but that when they shared the same modifier the different-relation primes were less effective. This is consistent with findings from studies of English and suggests that the properties of the modifier and head noun remain consistent regardless of their order in a combination. While our results agree with predictions of the CARIN theory, we speculate that this effect may be due to different senses of the modifier being appropriate depending on its associated relation. Consequently modifiers using different relations are less effective at priming targets with the same relation used in a different sense.

\section{Acknowledgements}

This research was funded by a UCD grant to the first author. We would like to thank Nicole Maguire for assistance in creating the French materials and we would also like to thank Rebecca Maguire for valuable comments and feedback.

\section{References}

Devereux, B. \& Costello, F. J. (2004). Learning relations between concepts: classification and conceptual combination. In Proceedings of the Twenty-Sixth Annual Conference of the Cognitive Science Society, (Chicago). Hillsdale, NJ: Erlbaum.

Gagné, C. L. (2001). Relation and lexical priming during the interpretation of noun-noun combinations. Journal of Experimental Psychology: Learning, Memory and Cognition, 27, 236-254.

Gagné, C. L., \& Shoben, E. J. (1997). The influence of thematic relations on the comprehension of modifier-noun combinations. Journal of Experimental Psychology: Learning, Memory and Cognition, 23, 71-87. 